\title{
Effects of tetraspanin CD151 inhibition on A549 human lung adenocarcinoma cells
}

\author{
PENGCHENG LI, HESONG ZENG, JIN QIN, YUANLIN ZOU, \\ DAN PENG, HOUJUAN ZUO* and ZHENGXIANG LIU*
}

\begin{abstract}
Departments of Cardiology and Internal Medicine, Tongji Hospital, Tongji Medical College, Huazhong University of Science and Technology, Wuhan, Hubei 430030, P.R. China
\end{abstract}

Received November 25, 2013; Accepted June 26, 2014

DOI: $10.3892 / \mathrm{mmr} .2014 .2774$

\begin{abstract}
Tetraspanin protein CD151 is overexpressed in a wide variety of cancer types, including lung cancer, and is closely associated with metastasis and poor prognosis of carcinoma. To investigate whether knockdown of CD151 expression can inhibit the malignant biological behavior of lung adenocarcinoma (LAC), RNA interference technology (RNAi) was used to silence CD151 expression in the A549 LAC cell line. Specific small interfering RNA (siRNA) for targeting human endogenous CD151 were delivered into A549 cells in order to examine the effects on cell proliferation, survival, migration, invasion and colony formation. The expression levels of CD151 were assayed by western blotting, proliferation was evaluated by MTT method and apoptosis was determined by flow cytometry. The invasive and metastatic ability of A549 cells was investigated by wound healing and Boyden chamber assays. Colony formation analysis was used to determine the A549 cell growth properties. Finally, the expression of phosphorylated FAK, PI3K-AKT, MEK-Erk1/2, MMPs, and VEGF was detected by western blotting. The results demonstrated that CD151-siRNA significantly decreased the expression level of CD151 in A549 cells. Reduced CD151 expression in A549 cells lead to the inhibition of cellular proliferation, migration, invasion and colony formation and an enhancement of apoptosis. Furthermore, the expression of tumor development-related proteins, including FAK, PI3K-AKT, MEK-ERK1/2MAPK as well as the expression of MMP9 and VEGF, were restrained. Taken
\end{abstract}

Correspondence to: Dr Houjuan Zuo or Dr Zhengxiang Liu, Departments of Cardiology and Internal Medicine, Tongji Hospital, Tongji Medical College, Huazhong University of Science and Technology, 1095 Jiefang Avenue, Wuhan, Hubei 430030, P.R. China E-mail: tuoye2004@163.com

E-mail: 477352186@qq.com

*Contributed equally

Key words: CD151, lung adenocarcinoma, small interference RNA, gene therapy together, the present study has shown that CD151 expression is essential for LAC progression. Thus, knockdown CD151 expression by targeted siRNA could inhibit the related downstream intercellular signaling pathways, and this may provide a novel gene therapy for patients with LAC.

\section{Introduction}

Lung cancer is one of the most common causes of cancerrelated mortalities in the world $(1,2)$. Lung adenocarcinoma (LAC) is one of the most common histological types of this disease, and its incidence has been gradually increasing worldwide $(3,4)$. Patients with LAC often experience chronic physical and psychological symptoms, including physical impairment, and the majority of patients succumb to the disease within 2 years (5). Therefore, there is an emergent requirement for novel interventions for the treatment of LAC.

CD151 is a member of the tetraspanin or transmembranefour superfamily (TM4SF), which was first discovered in a megakaryocyte cell line (MO7e) (6). It is frequently highly expressed in tumor cells. Regulating cell progression and movement is the most prominent feature of this protein (7-9). A number of previous studies have demonstrated that CD151 has a key role in cell proliferation, migration, colony formation and signaling transduction(10-12). Increased CD151 protein expression is associated with a poor prognosis in a number of different types of cancer. It has been shown that CD151 mediates positive effects on pro-carcinogenesis(13-15) and previous studies have indicated that inhibiting CD151 expression may significantly reduce cancer cell progression $(16,17)$. In benign prostatic hyperplasia, the level of CD151 expression has been reported to be very low, however the opposite has been observed in prostate cancer $(14,18)$. In addition, previous studies have detected that the expression level of CD151 was positively correlated with tumor progression, degree of tumor differentiation and clinical prognosis of lung cancer $(13,19)$. A positive correlation was also found in hepatocarcinoma, prostate cancer and colon cancer, demonstrating that CD151 may function in tumor progression and metastasis. An earlier study from our group had shown that the capacity of migration was significantly upregulated in prostate cancer cells that were transfected with CD151. This effect could be inhibited by mutations to CD151 (20). Thus, 
tetraspanin, and especially the critical role of CD151, may be a potential target for gene therapy in human LAC.

RNA interference (RNAi) refers to the degradation of homologous mRNA induced by double-stranded small interfering RNA, which is highly conserved throughout evolution. Through the use of RNAi technology it is possible specifically remove or turn off the expression of specific genes. RNAi technology has been used widely in recent years to explore gene function and the treatment of infectious diseases and cancer $(21,22)$. As such, RNAi has broad applications in the treatment of disease.

In the present study, siRNA was used to silence endogenous CD151 expression in A549 cells, to perturb the expression of downstream signaling molecules and the activation of specific cytokines. The experiments of this study analyzed the proliferation, migration, invasion and colony formation capacity of CD151-deficient A549 cells as well as the level of apoptosis. In addition, an investigation into the molecular mechanisms by which CD151 knockdown induces anti-tumor effects was performed. An understanding of the functional mechanism of CD151 in LAC may provide a novel approach for gene therapy in this disease.

\section{Materials and methods}

Materials. The human LAC cell line, A549, was purchased from the China Center for Type Culture Collection (Wuhan, China). Fetal bovine serum (FBS), cell culture medium, and TRIzol ${ }^{\circledR}$ reagent were purchased from Gibco-BRL (Carlsbad, CA, USA). Lipofectamine ${ }^{\mathrm{TM}} 2000$ reagent was purchased from Invitrogen Life Technologies (Carlsbad, CA, USA) for transfection of siRNAs obtained from Genepharma (Shanghai, China). MTT was purchased from Sigma-Aldrich. (St. Louis, MO, USA). The prestained molecular weight standards were obtained from Bio-Rad (Hercules, CA, USA), and polyvinylidene difluoride (PVDF) membranes from Schleicher and Schuell GmbH (Dassel, Germany). Borden chamber plates (Costar Corning, Cambridge, MA, USA) were prepared for the Matrigel invasion assay. The AnnexinV/fluorescein isothiocyanate (FITC) apoptosis detection kit was purchased from KeyGen Biotech Co. (Nanjing, China). Antibodies against CD151, PI3K, p-FAK, p-Akt, p-Erk1/2, p-MEK, VEGF, MEK, MMP-2, MMP-9 and $\beta$-actin were bought from Santa Cruz Biotechnology, Inc. (Santa Cruz, CA, USA). Antibodies against FAK and Akt were obtained from Cell Signaling Technology (Beverly, MA, USA). Enhanced chemiluminescence (ECL) reagent was purchased from Pierce Biotechnology, Inc. (Rockford, IL, USA). All other reagents were obtained from standard commercial suppliers unless otherwise specified.

Cell line and cell culture. The A549 cells were cultured in RPMI-1640 medium, supplemented with 10\% FBS, $100 \mathrm{U} / \mathrm{ml}$ penicillin, and $100 \mu \mathrm{g} / \mathrm{ml}$ streptomycin and incubated at $37^{\circ} \mathrm{C}$ in a humidified atmosphere containing $5 \% \mathrm{CO}_{2}$ and $95 \%$ air. Throughout the experiments, the cells were used in the logarithmic phase of growth.

Transfection of synthetic siRNA. The A549 cells were cultured in 6-well plates for $24 \mathrm{~h}$. Once the cells reached an $80 \%$ density/well, they were transfected with either 50, 75, and
$100 \mathrm{~nm}$ siRNA using Lipofectamine 2000 reagent, according to the manufacturer's instructions. The cells were harvested $24 \mathrm{~h}$ after transfection for analyses. A549 cells that had been either untreated or treated only with Lipofectamine 2000 reagent were used as controls.

Proliferation and apoptosis assay. The A549 cells were transfected with siRNA in 6-well plates, using Lipofectamine 2000. The cells were trypsinized $24 \mathrm{~h}$ following transfection, and seeded in 96 -well plates in triplicate $\left(1 \times 10^{4}\right.$ cells/well). Following adherence, the cells were exposed to RPMI-1640 medium with $0.5 \%$ FBS for $24 \mathrm{~h}$, and then the effects of downregulated CD151 on A549 cell proliferation was evaluated by MTT colorimetric assay. The medium was removed and replaced with medium containing $5 \mathrm{mg} / \mathrm{ml} \mathrm{MTT}$ and then incubated for $4 \mathrm{~h}$. The medium was aspirated, and the product was solubilized using dimethyl sulfoxide. Finally, the absorbance $(A)$ was measured at $490 \mathrm{~nm}$ for each well using a microplate reader (Bio Tek Instruments Inc., Winooski, VT, USA) according to the manufacturer's instructions. To measure the effects on apoptosis, cells were harvested $24 \mathrm{~h}$ after transfection and resuspended in binding buffer. FITC-conjugated Annexin V and propidium iodide (PI) (KeyGen Biotech Co.) was added to the cells, and all samples were incubated for $15 \mathrm{~min}$ at room temperature. Cells were then analyzed using a FACStar-Plus flow cytometer (BD Biosciences, Franklin Lakes, NJ, USA) to determine the percentage of apoptotic cells. The results were calculated using the percentage of apoptotic cells by the percentage of cells in the upper-right quadrant (Annexin V-positive, PI-positive) plus cells in the lower right quadrant (Annexin V-positive, PI-negative) to the total number of cells.

Western blotting. The A549 cells were incubated with radioimmunoprecipitation assay (RIPA) buffer containing $50 \mathrm{mmol} / \mathrm{l}$ Tris-HCl (pH 8.0), 0.5\% deoxycholic acid, $1 \%$ Nonidet-P40, $150 \mathrm{mmol} / 1 \mathrm{NaCl}$ and $0.1 \%$ sodium dodecyl sulfate for $30 \mathrm{~min}$ on ice. The cells were then detached from the plates using a cell scraper. The concentration of protein from the lysates of each group was obtained. Subsequently, $40 \mu \mathrm{g}$ protein was separated by SDS-PAGE and transferred to a PVDF membrane. The PVDF membranes were blocked for $2 \mathrm{~h}$ at room temperature with $5 \%$ fat-free dried milk in tris-buffered saline with tween (10 mm Tris- $\mathrm{HCl}, 100 \mathrm{~mm} \mathrm{NaCl}$, and $0.1 \%$ Tween-20). The membranes were then incubated with primary antibodies overnight at $4^{\circ} \mathrm{C}$, followed by secondary antibodies against rabbit or mouse $\mathrm{IgG}$ conjugated to horseradish peroxidase (1:3000) for $2.5 \mathrm{~h}$ at room temperature. Finally, ECL was used to detect the intensities of the various protein bands, which were quantified by densitometry. $\beta$-actin FAK, t-AKT, MEK and ERK were used as loading controls.

Migration and invasion assay. A549 cells were transfected with siRNAs in 6-well plates. Following $24 \mathrm{~h}$ transfection, when the cells reached $90 \%$ confluency/well, the cells were scratched with a $200 \mu$ l pipette tip. The plates were then washed twice with phosphate-buffered saline to remove the detached cells and incubated in FBS-free medium. Cells that migrated into the wounded area were imaged immediately $(0 \mathrm{~h})$ and at $24 \mathrm{~h}$. The invasion assay was performed using a transwell 
chamber with $8 \mu \mathrm{m}$ pore size polycarbonate membrane (Costar Corning), using serum-free RPMI-1640 media diluted extracellular matrix (ECM) to coat the membrane. The A549 cells transfected with siRNA were trypsinized and seeded in the upper compartment of the upper wells at a density of $1 \times 10^{4}$ cells/well in $200 \mu \mathrm{l}$ of the serum-free media. The lower chamber was filled with media containing $20 \%$ FBS. Subsequently, the cells were able to invade the membrane for $24 \mathrm{~h}$. Following this, the non-migrated cells were removed from the upper surface of the polycarbonate membrane, and the invaded cells were stained with gentian violet. Invasion analysis was determined by counting the invaded cells under a microscope. Six visual fields were chosen at random from each of the weels and the number of the invaded cells in the six fields was averaged.

Clonogenic assay. Cells were seeded in 6-well plates at a concentration of 200-300 cells/well. Following transfection for $24 \mathrm{~h}$, the cells were incubated for 14 days at $37^{\circ} \mathrm{C}$ in a humidified atmosphere of $5 \% \mathrm{CO}_{2}$. The colonies were fixed in $4 \%$ paraformaldehyde at room temperature for $30 \mathrm{~min}$, stained with $0.1 \%$ crystal violet for $10 \mathrm{~min}$. At the end of the experiment, the positive colonies formed ( $>50$ cells/colony) were counted and the colony formation rate was calculated. This experiment was repeated three times.

Statistical analysis. All the experiments were repeated at least three times, and the results are expressed as the mean \pm standard error of the mean. Comparisons between the two groups were performed by Fisher $t$-test and those among three groups were followed by one-way analysis of variance. A value of $\mathrm{P}<0.05$ was considered to indicate a statistically significant difference.

\section{Results}

CD151-siRNA targets the endogenous CD151 gene and effectively inhibits its protein expression in A549 cells in vitro. To determine whether CD151 protein was effectively decreased in A549 cells following siRNA transfection, the protein expression was examined by western blot analyses. The degree of CD151 protein inhibition in A549 cell lines was different according to different doses of siRNA. The protein inhibition occurred in a dose-depended manner. A549 cells that had been either untreated or treated only with Lipofectamine 2000 reagent were used as controls. The results of the western blot analysis revealed that the CD151 protein was significantly downregulated in A549 cells as compared with the control and mock group $(\mathrm{P}<0.05)$ (Fig. 1). When a $100 \mathrm{nM}$ dose of siRNA was used, the transfection efficiency was at its highest.

CD151 gene inhibition directly decreases the proliferation rate of A549 cells, but the percentage of apoptotic cells in the siRNA treated group is increased. To investigate whether endogenous CD151 inhibition affected the proliferation and apoptosis of A549 cells, cells were transfected with CD151 siRNA and $24 \mathrm{~h}$ after transfection, the proliferation rates were determined by MTT assay. As shown in Fig. 2A, the growth rate of CD151-siRNA treated A549 cells was significantly decreased after 24, 48 and $72 \mathrm{~h}(\mathrm{P}<0.05$, respectively), when compared to the control and mock cells. No significant difference was observed between the
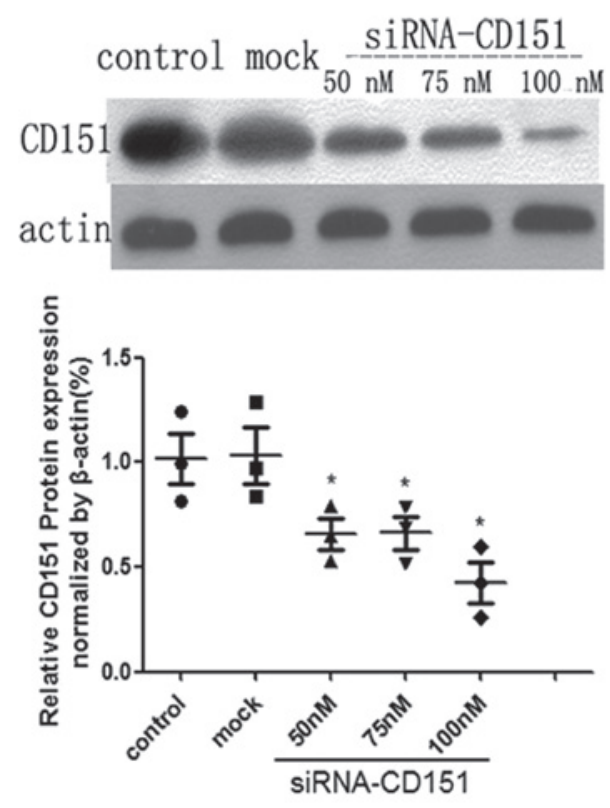

Figure 1. Expression levels of CD151 protein in A549 cells under different doses of CD151-siRNA. The cellular proteins were extracted in lysis buffer and CD151 protein levels were examined by western blotting. The $\beta$-actin protein levels were detected as a control. The data points represent the mean of three experiments and the error bars represent the standard deviation. ${ }^{*} \mathrm{P}<0.05$ vs control and mock. All data shown were repeated three times. siRNA, small interfering RNA.

control and mock groups. These data demonstrated that knockdown of CD151 may have a significant negative effect on the proliferation of A549 cells. Apoptosis, measured by Annexin V and PI staining, was significantly increased in cells treated with CD151-siRNA (Fig. 2B). There was no significance difference between the control and mock group cells. These data suggested that knockdown of CD151 expression resulted in decreased proliferation and activated apoptosis in A549 cell lines.

Targeting endogenous CD151 siRNA weakens the migratory/invasive ability of A549 cells. CD151 is associated with the biological behavior of tumor cells including metastasis, for which two critical steps are migration and invasion. To investigate the effects of CD151 on the metastasis of A549 cells, siRNA was used to silence the endogenous CD151 expression in A549 cells and wound healing migration and Boyden chamber invasion assays were performed. In the wound healing assay, CD151 siRNA-treated cells exhibited a significantly decreased migration rate and were unable to repair the wound as compared with the scrambled siRNA mock cells. The control and mock cells achieved almost complete wound healing (Fig. 3A). Furthermore, in the Boyden chamber assay, the number of invasive cells among those treated with CD151 siRNA was significantly decreased as compared to the mock cells. These results indicated that CD151 inhibition decreased the invasive capacity of A549 cells, but there was no significant difference between the control and mock cells (Fig. 3B). These data showed that targeting of CD151 siRNA weakens the migratory and invasive abilities of A549 cells in vitro.

Inhibition of colony formation in CD151 knockdown A549 cells. To further study the role of CD151 inhibition on 
A

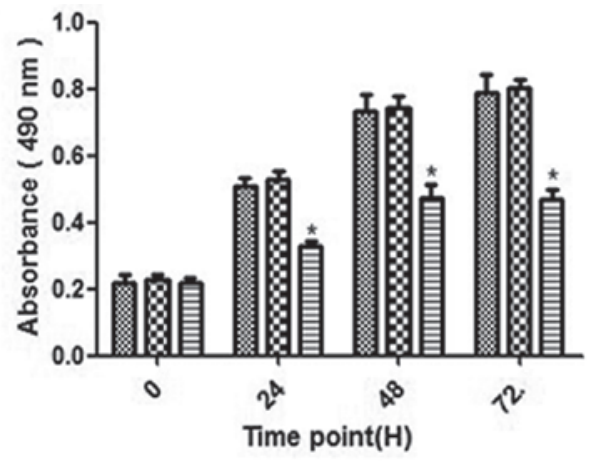

B

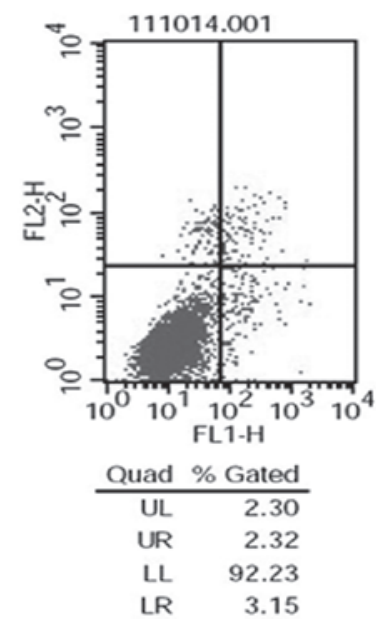

mock

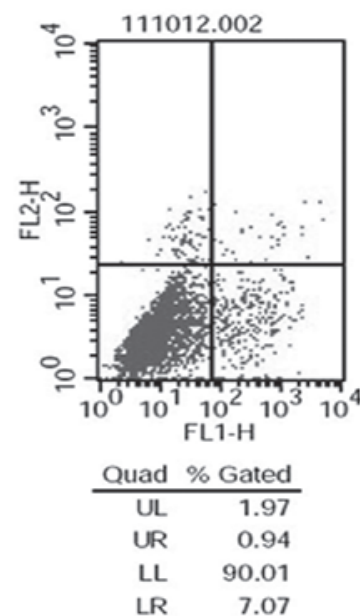

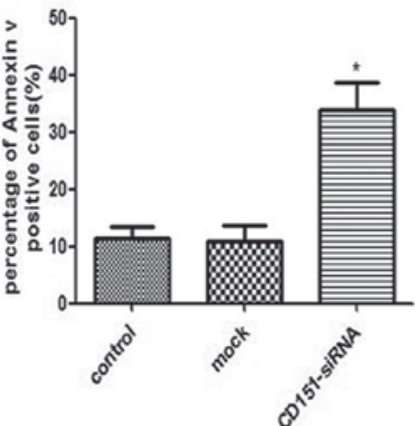

CD151-siRNA

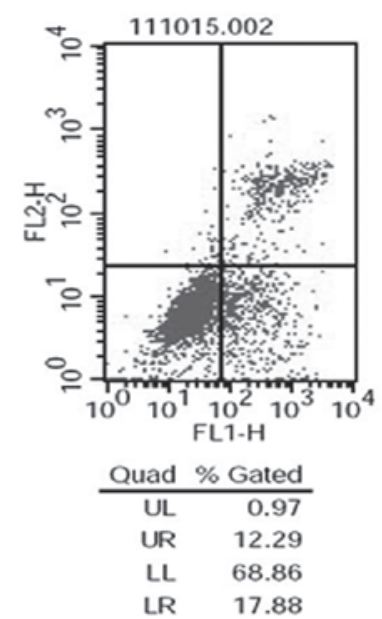

Figure 2. Inhibition of the endogenous CD151 protein expression with CD151-siRNA significantly inhibits A549 cell proliferation, but the apoptosis rate of A549 cells was directly increased. (A) A549 cells were seeded into 96-well plates and transfected with CD151 and scrambled siRNAs (mock) at $100 \mathrm{~nm}$. Untreated cells were used as a control. The three groups of cells were incubated at $37^{\circ} \mathrm{C}$ in a humidified atmosphere containing $5 \% \mathrm{CO}_{2}$ and $95 \%$ air for $0-72 \mathrm{~h}$ and were analyzed for $0,24,48$, and $72 \mathrm{~h}$ by MTT assay. CD151-siRNA-treated A549 cell growth was significantly inhibited at 24,48 and $72 \mathrm{~h}(\mathrm{P}<0.05)$ as compared with the mock cells. (B) A549 cells were seeded in 6-well plates and transfected with CD151-siRNA for 24 h. The cells stained with Annexin V/ fluorescein isothiocyanate and propidium iodide were used for subsequent analysis by flow cytometry. The percentage of apoptotic cells was increased in CD151-siRNA treated cells, whereas there was no increase in the cells treated with scrambled siRNA sequence. The percentage of cells in the upper-right quadrant (propidium iodide-positive, Annexin V-positive) plus the cells in the lower-right quadrant (propidium iodide-negative, Annexin V-positive) from the total cell number are represented under the relevant graph. The columns represent the mean of three experiments and the error bars represent the standard deviation. "P<0.05 vs control and mock. UL, upper left; UR, upper right; LL, lower left; LR, lower right; siRNA, small interfering RNA.

the growth properties of A549 cells, a colony formation ( $>50$ cells/colony) assay was performed. Control, mock and CD151-siRNA cells were seeded at a density of 300 cells $/ \mathrm{ml}$ in 6-well plates. Following $24 \mathrm{~h}$ transfection, the cells were incubated at $37^{\circ} \mathrm{C}$ in a humidified atmosphere of $5 \% \mathrm{CO}_{2}$ for 14 days. Finally, positive colonies were counted. As shown in Fig. 4, siRNA-treated cells exhibited a significantly lower colony formation rate as compared with the control and mock cells. There was no significant difference between the control and mock group. CD151 downregulation resulted in a marked inhibition in the capacity of A549 cells to form colonies.

Molecular mechanisms by which CD151 knockdown exhibits the anti-tumor effects. Protein samples from the three groups of cells were extracted to investigate the role of the CD151 knockdown in the expression and phosphorylation changes of signaling molecules and specific cytokines. It was observed that the protein expression levels of phosphorylated FAK, PI3K, phosphorylated Akt, phosphorylated MEK, and phosphorylated Erk1/2, were downregulated in the CD151
siRNA-treated cells, whereas those in the control and mock cells had no significant difference (Fig. 5A and B). These results demonstrated that $\mathrm{CD} 151$ inhibition had significantly decreased expression of intracellular growth factor signaling molecules in A549 cells. In addition, CD151 knockdown also disturbed the protein expression of VEGF and MMP-9, which have been documented to directly promote carcinogenesis and tumor progression $(23,24)$. However, a difference in expression of MMP-2 protein was not observed (Fig. 5C). Together, these data indicated that CD151 knockdown in A549 cells functions in anti-tumor effects through the inhibition of downstream proliferation and metastasis pathways.

\section{Discussion}

Despite the advances in medical and surgical treatments, lung cancer remains the leading cause of cancer-related death (2). Because of the intrinsic properties of LAC and the ability of tumor cells to rapidly progress, the prognosis in LAC is poor. Tumor progression involves tumor cell proliferation, 


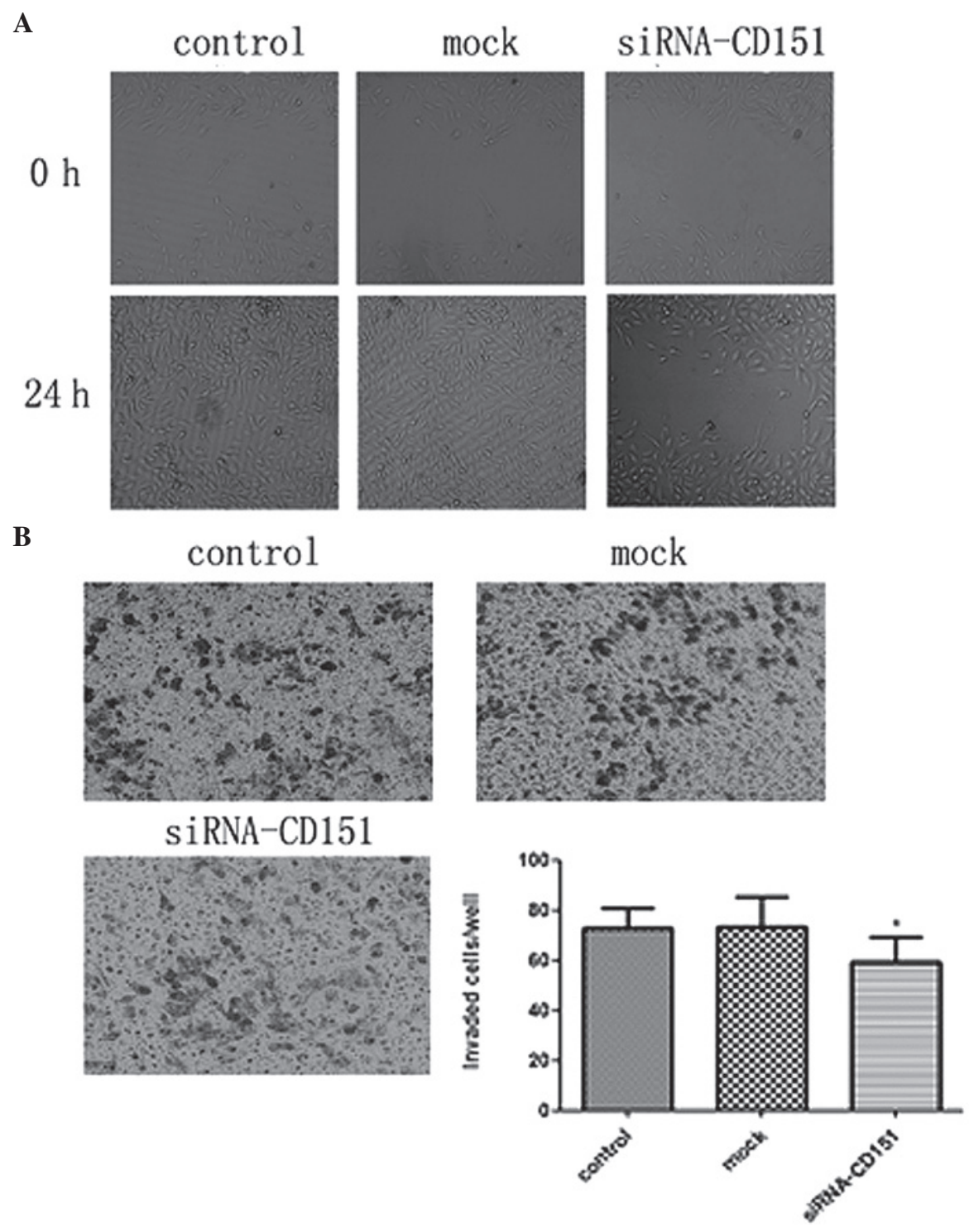

Figure 3. Inhibition of CD151 expression markedly decreases the migration and invasion capacity of A549 cells. (A) A549 cells were seeded and transfected in 6-well plates for $24 \mathrm{~h}$, and after reaching $~ 90 \%$ confluency, the cells were dislodged using a $200 \mu \mathrm{l}$ pipette tip. The cells were then incubated in fetal bovine serum-free medium for $24 \mathrm{~h}$, following which images were taken. Data and representative images are shown at x200 magnification. A549 cells treated with CD151-siRNA exhibited a marked decrease in cell motility. (B) Cells were harvested after $24 \mathrm{~h}$ transfection, and then $100 \mu 1$ homogeneous single cell suspension was added to the Matrigel-coated Transwell upper chambers. Representative microscopy images are shown at x200 magnification. CD151-siRNA significantly inhibited the invasion ability of A549 cells, as compared with the number of invading cells in the control groups. The columns represent the mean of three experiments and the error bars represent the standard deviation. ${ }^{*} \mathrm{P}<0.05$ vs control and mock. siRNA, small interfering RNA.
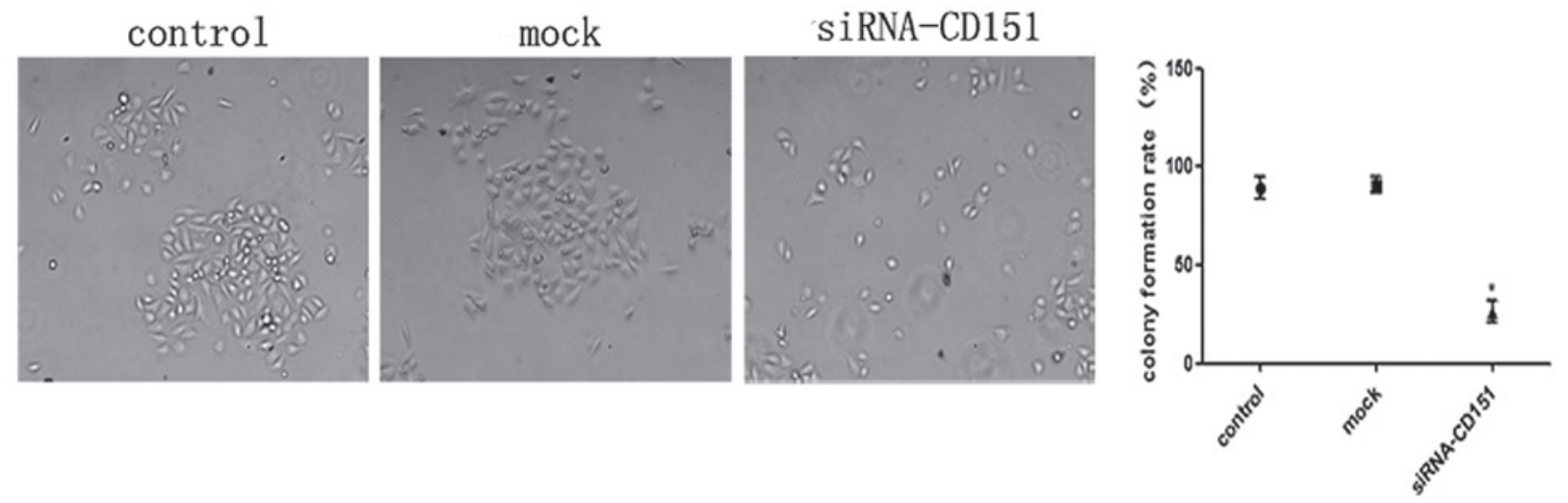

Figure 4. CD151 knockdown decreases colony formation of A549 cells. Cells transfected with CD151-siRNA were seeded in 6-well plates at a density of 300 cells per well. After 14 days incubation, the positive colony formations ( $>50$ cells/colony) were counted. Representative microscopy images are shown (magnification, x200). The points represent the mean of three experiments and the error bars represent the standard deviation. "P<0.05 vs control and mock. siRNA, small interfering RNA. 
A
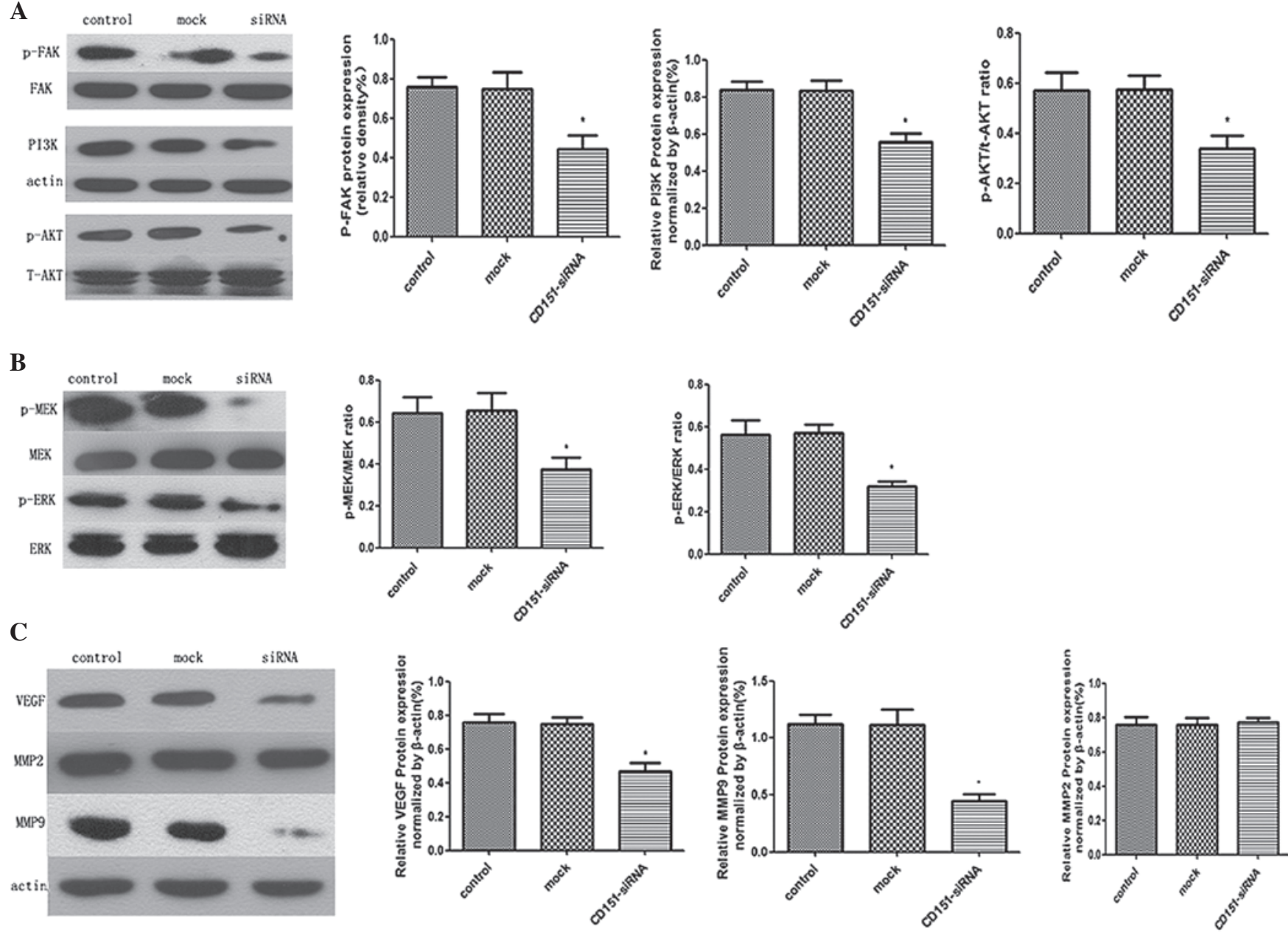

Figure 5. Western blot analysis of the anti-tumor effects of CD151 knockdown using siRNA in A549 cells. A549 cells were treated with CD151-siRNA or scrambled siRNA for $24 \mathrm{~h}$, and then total cell protein was extracted. (A and B) The levels of phosphorylated FAK, PI3K, phosphorylated Akt, phosphorylated MEK and phosphorylated Erk1/2 were examined by western blotting. CD151-siRNA-treated A549 cells exhibited decreased activation of FAK, PI3K/Akt and MEK/Erk1/2 MAPK as compared with the control and mock group. (C) The effects of CD151 downregulation on MMPs and VEGF expression. The MMP-9 and VEGF protein expression in CD151-siRNA-treated A549 cells was relatively diminished as compared with the control and mock group. There was no difference in the protein expression level of MMP-2 among these three groups. The columns represent the mean of three experiments and the error bars represent the standard deviation. "P<0.05 vs control and mock. MMP, matrix metalloproteinase; FAK, focal adhesion kinase; PI3K, phosphoinositide 3-kinase; MEK, mitogen-activated protein kinase kinase; ERK, extracellular signal-regulated kinase; VEGF, vascular endothelial growth factor; p, phosphorylated; siRNA, small interfering RNA.

migration, invasion and vascular intravasation or extravasation, establishment of a metastatic niche, and angiogenesis $(25,26)$. Therefore, controlling the biological behavior of LAC cells in proliferation, migration and invasion ability is crucial for LAC therapy.

CD151 is a member of the tetraspanins, transmembrane 4 superfamily (TM4SF). It has been considered to have important roles in various cellular functions, including cell proliferation, differentiation, adhesion, and motility (16). Previous studies have indicated that CD151 is highly expressed in numerous cancers, thus contributing to the malignancy of human carcinoma. Overexpression of CD151 clearly induced tumorigenicity $(16,27,28)$. Data has shown that CD151 overexpression enhanced the invasive and metastatic capability of numerous cancer cell lines, however, the treatment of cells with anti-CD151 suppressed this ability (10). Anti-CD151 antibodies or CD151 mutants could selectively inhibit integrin- $\alpha 3 / \alpha 6$-dependent cell metastasis, whereas upregulation of CD151 enhanced experimental migration of colon carcinoma and fibrosarcoma cells (24). Although the biochemical functions of CD151 remain elusive, the ability of tetraspanin proteins to associate with each other and other membrane proteins demonstrates that CD151 may have a role as a transmembrane connector that links intercellular molecular signaling. It has been reported that in various normal cells, CD151 controls intercellular signaling balance and regulates cell survival, proliferation, movement, differentiation and morphology (29). Nevertheless, under pathological conditions, CD151 may promote tumor progression on account of its ability to promote cell survival, proliferation, movement, colony formation and signaling transduction. All of its abilities may significantly alter the cellular environment, and consequently result in tumor progression. However, the definitive role of CD151 in cancer progression and metastasis remains to be determined, and it remains undetermined whether CD151 inhibition has an important role in LAC..

In the present study, siRNA was used for targeted dowregulation of CD151 expression in the LAC cell line, A549, to investigate a novel anti-tumor gene therapy. CD151-siRNAs were synthesized to specifically target endogenous CD151 in 
A549 cells, according to the literature (30). The transfection efficiency was $\sim 75 \%$ and the siRNA efficacy was sustained for $>24 \mathrm{~h}$ without degradation. Furthermore, western blot analysis demonstrated that the efficiency of gene silencing occurred in a dose-dependent manner. When the concentration of CD151-siRNA was at $100 \mathrm{~nm}$, the transfection efficacy was highest. This method of siRNA-induced gene silencing was appropriate based on the specificity of the siRNA designed against CD151. It was identified that inhibiting the expression of CD151 resulted in restraint of proliferation in A549 cells, in a time-dependent manner. Furthermore, tumor colony-forming analysis indicated that the percentage of positive colonies formed following CD151 knockdown in A549 cells was significantly reduced. The percentage of apoptotic A549 cells following CD151-siRNA transfection was significantly increased. Therefore, knockdown of CD151 expression effectively diminished the capacity of A549 cell survival and colony formation. Moreover, we investigated the signaling pathways related to cell growth, including FAK, and other independent pathways, including PI3K/AKT and MEK-ERK/MAPK. The results indicated that knockdown of CD151 expression in A549 LAC cells, led to a decrease in FAK, PI3K/Akt, MEK/Erk1/2 phosphorylation. There was no significant difference between the control and mock cells. The chemically synthesized CD151-siRNAs targeting CD151 may therefore restrain the uncontrolled growth of A549 cells and promote cell apoptosis through downstream intercellular signaling molecules.

Beyond participating in the growth of A549 cells, it was identified that the capabilities of metastasis and invasion were impaired in cells undergoing CD151-siRNA treatment. This was consistent with our previous hypothesis that CD151 is required for LAC metastasis and invasion, similar to other cancer types (13). Tetraspanin proteins have been considered to be associated with signal transduction by modulating the organization and assembly of signaling complexes in membrane microdomains, known as the 'tetraspanin web' (31-33). CD151 has a strong lateral association with laminin-banding integrins, including $\alpha 3 \beta 1, \alpha 6 \beta 1, \alpha 6 \beta 4$ and $\alpha 7 \beta 1$. In contrast to previously confirmed roles of CD151 as an adaptor for integrin-delivered signaling molecular, some previous research has indicated that CD151 can modulate its own signals such as upregulation of MMPs, cell motility, and invasiveness. Although it has been demonstrated that increased expression of MMP-2 and MMP-9 function in lung cancer in cell invasion and metastasis (24), the manner in which CD151 modulates MMPs in LAC remains elusive. To the best of our knowledge, the present study confirmed for the first time that CD151 knockdown markedly decreased the expression level of MMP9 but not MMP2. In addition, angiogenesis also functions in LAC progression and metastasis and VEGF has a key function in the process of neovascularization. It was identified that VEGF expression was significantly decreased in A549 cells treated with CD151-siRNA. Thus, it was hypothesized that CD151 may directly regulate MMP-9 and VEGF expression in A549 cells and promote tumor progression and invasion, however, the molecular mechanism remains to be discovered.

In summary, the results of the present study have confirmed that downregulation of CD151 by targeted siRNA inhibited the proliferation, metastasis, invasion and colony formation, but induced apoptosis in A549 cells. Downregulation of CD151 by siRNA may be a novel method for gene therapy in LAC. Further studies are warranted to verify the effectiveness and safety for anti-LAC therapy in vivo.

\section{Acknowledgements}

The study was supported by the National Natural Science Foundation of China (nos. 81000047 and 81000139).

\section{References}

1. Bennett A and White J: Improving care and quality of life for patients with lung cancer. Nurs Stand 28: 50-58, 2013.

2. Jemal A, Siegel R, Xu J, et al: Cancer statistics, 2010. CA Cancer J Clin 60: 277-300, 2010.

3. Oken MM, Hocking WG, Kvale PA, et al: Screening by chest radiograph and lung cancer mortality: the Prostate, Lung, Colorectal, and Ovarian (PLCO) randomized trial. JAMA 306: 1865-1873, 2011.

4. Hanagiri T, Baba T, So T, et al: Time trends of surgical outcome in patients with non-small cell lung cancer. J Thorac Oncol 5: 825-829, 2010.

5. Sun X and Zheng Y: Retreatment with icotinib in a patient with metastatic lung adenocarcinoma. Tumori 99: e124-e126, 2013.

6. Hashida H, Takabayashi A, Tokuhara T, et al: Clinical significance of transmembrane 4 superfamily in colon cancer. Br J Cancer 89: 158-167, 2003.

7. Kang BW, Lee D, Chung HY, et al: Tetraspanin CD151 expression associated with prognosis for patients with advanced gastric cancer. J Cancer Res Clin Oncol 139: 1835-1843, 2013.

8. Wang HX, Li Q, Sharma C, et al: Tetraspanin protein contributions to cancer. Biochem Soc Trance 39: 547-552, 2011.

9. Yue S, Mu W and Zöller M: Tspan 8 and CD151 promote metastasis by distinct mechanisms. Eur J Cancer 49: 2934-2948, 2013.

10. Copeland BT, Bowman MJ and Ashman LK: Genetic ablation of the tetraspanin CD151 reduces spontaneous metastatic spread of prostate cancer in the TRAMP model. Mol Cancer Res 11: 95-105, 2013.

11. Chernousov MA, Stahl RC and Carey DJ: Tetraspanins are involved in Schwann cell-axon interaction. J Neurosci Res 91: 1419-1428, 2013.

12. Spring FA, Griffiths RE, Mankelow TJ, et al: Tetraspanins CD81 and CD 82 facilitate $\alpha 4 \beta 1$-mediated adhesion of human erythroblasts to vascular cell adhesion molecule-1. PLoS One 8: e62654, 2013.

13. Kwon MJ, Seo J, Kim YJ, et al: Prognostic significance of CD15 overexpression in non-small cell lung cancer. Lung Cancer 81: 109-116, 2013.

14. Ang J, Lijovic M, Ashman LK, et al: CD151 protein expression predicts the clinical outcome of low-grade primary prostate cancer better than histologic grading: a new prognostic indicator? Cancer Epidemiol Biomarkers Prev 13: 1717-1721, 2004.

15. Lee D, Suh YL, Park TI, et al: Prognostic significance of tetraspanin CD151 in newly diagnosed glioblastomas. J Surg Oncol 107: 646-652, 2013.

16. Haeuw JF, Goetsch L, Bailly C, et al: Tetraspanin CD151 as a target for antibody-based cancer immunotherapy. Biochem Soc Trans 39: 553-558, 2011

17. Minner S, De Silva C, Rink M, et al: Reduced CD151 expression is related to advanced tumour stage in urothelial bladder cancer. Pathology 44: 448-452, 2012.

18. Ang J, Fang BL, Ashman LK, et al: The migration and invasion of human prostate cancer cell lines involves CD151 expression. Onco Rep 24: 1593-1597, 2010.

19. Woegerbauer M, Thurnher D, Houben R, et al: Expression of the tetraspanins CD9, CD37, CD63, and CD151 in Merkel cell carcinoma: strong evidence for a posttranscriptional fine-tuning of CD9 gene expression. Mod pathol 23: 751-762, 2010.

20. Yang W, Li P, Lin J, et al: CD151 promotes proliferation and migration of PC3 cells via the formation of CD151-integrin $\alpha 3 / \alpha 6$ complex. J Huazhong Univ Sci Technolog Med Sci 32: 383-388, 2012.

21. Gottumukkala SN, Dwarakanath CD and Sudarsan S: Ribonucleic acid interference induced gene knockdown. J Indian Soc Periodontol 17: 417-422, 2013. 
22. Wilson RC and Doudna JA: Molecular mechanisms of RNA interference. Annu Rev Biophys 42: 217-239, 2013.

23. Fujishima S, Shiomi T, Yamashita S, et al: Production and activation of matrix metalloproteinase 7 (matrilysin 1) in the lungs of patients with idiopathic pulmonary fibrosis. Arch Pathol Lab Med 134: 1136-1142,2010.

24. Hong IK, Jin YJ, Byun HJ, et al: Homophilic interactions of Tetraspanin CD151 up-regulate motility and matrix metalloproteinase-9 expression of human melanoma cells through adhesion-dependent c-Jun activation signaling pathways. J Biol Chem 281: 24279-24292, 2006.

25. Ogorevc E, Kralj-Iglic V and Veranic P: The role of extracellular vesicles in phenotypic cancer transformation. Radiol Oncol 47: 197-205, 2013.

26. Olbryt M: Role of tumor microenvironment in the formation and progression of skin melanoma. Postepy Hig Med Dosw (Online) 67: 413-432, 2013 (In Polish).

27. Wang HX, Li Q, Sharma C, et al: Tetraspanin protein contributions to cancer. Biochem Soc Trans 39: 547-552, 2011.
28. Romanska HM and Berditchevski F: Tetraspanins in human epithelial malignancies. J Pathol 223: 4-14, 2011.

29. Takeda Y, Kazarov AR, Butterfield CE, et al: Deletion of tetraspanin $\mathrm{Cd} 151$ results in decreased pathologic angiogenesis in vivo and in vitro. Blood 109: 1524-1532, 2007.

30. Yañez-Mó M, Barreiro O, Gonzalo P, et al: MT1-MMP collagenolytic activity is regulated through association with tetraspanin CD151 in primary endothelial cells. Blood 112: 3217-3226, 2008.

31. Devbhandari RP, Shi GM, Ke AW, et al: Profiling of the tetraspanin CD151 web and conspiracy of CD151/integrin $\beta 1$ complex in the progression of hepatocellular carcinoma. PLoS One 6: e24901, 2011.

32. Colin S, Guilmain W, Creoff E, et al: A truncated form of CD9-partner 1 (CD9P-1), GS-168AT2, potently inhibits in vivo tumour-induced angiogenesis and tumour growth. Br J Cancer 105: 1002-1011, 2011.

33. Charrin S, Manié S, Billard M, et al: Multiple levels of interactions within the tetraspanin web. Biochem Biophys Res Commun 304: 107-112, 2003. 